\title{
О регулярных тканях, определенных плюригармоническими функциями
}

\author{
Л. М. Пиджакова, А. М. Шелехов
}

\begin{abstract}
As is known, the function of two variables $z=f(x, y)$ on the plane of the variables $(x, y)$ in the neighborhood of a regular point defines a three-web formed by the foliations $x=$ const, $y=$ const, and $f(x, y)=$ const. A three-web is called regular if it is equivalent (locally diffeomorphic) to a three-web formed by three families of parallel lines. In this case, the equation of the three-web has the form $z=f(\alpha(x)+\beta(y))$.

In one of the works of the authors of this article, all regular three-webs, determined by some well-known partial differential equations, in particular, determined by harmonic functions, were found. In the present paper, the results are generalized for pluriharmonic functions of the form
\end{abstract}

$$
u=f\left(x_{1}, \ldots, x_{r}, y_{1}, \ldots, y_{r}\right) .
$$

First, a function of this type on a manifold of dimension $2 r$ determines a $(2 r+1)$-web formed by foliations of codimension 1 :

$$
x_{i}=\text { const }, \quad y_{i}=\text { const },(i=1,2, \ldots, r), \quad u=\text { const. }
$$

A $(2 r+1)$-web is called regular if in some local coordinates its equation can be written as

$$
u=f\left(\varphi_{1}\left(x_{1}\right)+\ldots+\varphi_{r}\left(x_{r}\right)+\psi_{1}\left(y_{1}\right)+\ldots+\psi_{r}\left(y_{r}\right)\right) .
$$

In the present paper, we find all pluriharmonic functions defining regular $(2 r+1)$-webs (Theorem 1).

On the other hand, each pluriharmonic function

$$
u=f\left(x_{1}, \ldots, x_{r}, y_{1}, \ldots, y_{r}\right)
$$

defines a three-web $W(r, r, 2 r-1)$ on a $2 r$-dimensional manifold formed by two $r$-dimensional foliations $x_{i}=$ const and $y_{i}=$ const and one foliation $u=$ const of codimension 1 . This three-web is called regular if its equation in some local coordinates can be written as

$$
u=f\left(\varphi\left(x_{1}, x_{2}, \ldots, x_{r}\right)+\psi\left(y_{1}, y_{2}, \ldots, y_{r}\right)\right) .
$$

In this work found all pluriharmonic functions defining regular $W(r, r, 2 r-1)$ webs (Theorem 2).

Ключевые слова: плюригармоническая функция, регулярная ткань 
Анотація. Як відомо, функція двох змінних $z=f(x, y)$ визначає на площині $(x, y)$ в околі регулярної точки деяку три-тканину, утворену шаруваннями $x=$ const, $y=$ const та $f(x, y)=$ const. Три-тканина називається регулярною, якщо вона еквівалента (тобто локально дифеоморфна) три-тканині, утвореній трьома сім'ями паралельних прямих. В цьому випадку рівняння тканини має вигляд $z=f(\alpha(x)+\beta(y))$.

В одній з робіт авторів цієї статті були знайдені всі регулярні тритканини, що задаються деякими відомими рівняннями в частинних похідних, зокрема, визначаються гармонійними функціями. В даній роботі ці результати узагальнюються на випадок плюрігармонічних функцій виду $u=f\left(x_{1}, \ldots, x_{r}, y_{1}, \ldots, y_{r}\right)$.

По-перше, така функція на многовиді розмірності $2 r$ визначає $(2 r+1)$ тканину, утворену шаруваннями ковиміру 1 виду

$$
x_{i}=\text { const }, \quad y_{i}=\text { const },(i=1,2, \ldots, r), \quad u=\text { const. }
$$

$(2 r+1)$-тканина називається регулярною, якщо в деяких локальних координатах її рівняння може бути записано у вигляді

$$
u=f\left(\varphi_{1}\left(x_{1}\right)+\ldots+\varphi_{r}\left(x_{r}\right)+\psi_{1}\left(y_{1}\right)+\ldots+\psi_{r}\left(y_{r}\right)\right) .
$$

В представленій роботі ми знаходимо всі плюригармонічні функції, що визначають регулярні $(2 r+1)$-тканини (теорема 1$)$.

3 іншого боку, кожна плюригармонічна функція

$$
u=f\left(x_{1}, \ldots, x_{r}, y_{1}, \ldots, y_{r}\right)
$$

на $2 r$-вимірному многовиді визначає три-тканину $W(r, r, 2 r-1)$, утворену двома $r$-вимірними шаруваннями $x_{i}=$ const i $y_{i}=$ const та шаруванням $u=$ const ковиміру 1 . Ця тканина називається регулярною, якщо в деяких локальних координатах їі рівняння може бути записано у вигляді

$$
u=f\left(\varphi\left(x_{1}, x_{2}, \ldots, x_{r}\right)+\psi\left(y_{1}, y_{2}, \ldots, y_{r}\right)\right) .
$$

Ми також знаходимо всі плюригармонічні функції, що визначають регулярні три-тканини $W(r, r, 2 r-1)$ (теорема 2$)$.

В работе [3] рассматриваются решения вида $z=f(\alpha(x)+\beta(y))$ некоторых уравнений в частных производных. В частности, найдены гармонические функции такого строения. На плоскости $(X O Y)$ указанные функции определяют, как известно [1], [4], регулярные три-ткани.

Целью данной работы является обобщение полученных результатов для плюригармонических функций вида

$$
u=f\left(x_{1}, \ldots, x_{r}, y_{1}, \ldots, y_{r}\right) .
$$

$\mathrm{C}$ одной стороны, функция (1) определяет в окрестности регулярной точки пространства $\mathbb{R}^{2 r}$ переменных $\left(x_{i}, y_{i}\right)(2 r+1)$-ткань, образованную $2 r+1$ слоениями коразмерности $1: x_{i}=$ const, $y_{i}=$ const и $u=$ const (здесь и далее $i, j, k=1,2, \ldots, r)$. Уравнение (1) называется уравнением этой ткани. $(2 r+1)$-ткань называется регулярной, если допустимой 
заменой переменных ее уравнение можно привести к виду

$$
u=f\left(\varphi_{1}\left(x_{1}\right)+\ldots+\varphi_{r}\left(x_{r}\right)+\psi_{1}\left(y_{1}\right)+\ldots+\psi_{r}\left(y_{r}\right)\right) .
$$

$\mathrm{C}$ другой стороны, уравнение (1) определяет в пространстве $\mathbb{R}^{2 r}$ триткань $W(r, r, 2 r-1)$ со слоениями различной размерности: два $r$-параметрических слоения $x_{i}=$ const и $y_{i}=$ const, и одно слоение $u=$ const коразмерности 1. Три-ткань $W(r, r, 2 r-1)$ называется регулярной, если функция (1) имеет вид

$$
u=f\left(\varphi\left(x_{1}, x_{2}, \ldots, x_{r}\right)+\psi\left(y_{1}, y_{2}, \ldots, y_{r}\right)\right),
$$

или короче $u=f\left(\varphi\left(x_{i}\right)+\psi\left(y_{i}\right)\right)$.

Функция вида (1) называется плюригармонической, если выполняются следующие условия [2]:

$$
\begin{gathered}
\frac{\partial^{2} u}{\partial x_{i} \partial x_{j}}+\frac{\partial^{2} u}{\partial y_{i} \partial y_{j}}=0 \\
\frac{\partial^{2} u}{\partial x_{i} \partial y_{j}}=\frac{\partial^{2} u}{\partial x_{j} \partial y_{i}} .
\end{gathered}
$$

1. Найдём плюригармонические функции вида (2). Для них уравнения (5) примут вид:

$$
u^{\prime \prime} \cdot\left(\varphi_{i}^{\prime} \cdot \psi_{j}^{\prime}-\varphi_{j}^{\prime} \cdot \psi_{i}^{\prime}\right)=0
$$

а уравнения (4) - вид

$$
u^{\prime \prime} \cdot\left(\varphi_{i}^{\prime} \cdot \varphi_{j}^{\prime}+\psi_{i}^{\prime} \cdot \psi_{j}^{\prime}\right)=0 \text { при } i \neq j,
$$

и

$$
u^{\prime \prime} \cdot\left(\left(\varphi_{i}^{\prime}\right)^{2}+\left(\psi_{i}^{\prime}\right)^{2}\right)+u^{\prime} \cdot\left(\varphi_{i}^{\prime \prime}+\psi_{i}^{\prime \prime}\right)=0 \text { при } i=j .
$$

а) Если $u^{\prime \prime}=0$, то уравнения (6) и (7) удовлетворяются, а функция $u$ является линейной:

$$
u=a\left(\varphi_{1}\left(x_{1}\right)+\ldots+\varphi_{r}\left(x_{r}\right)+\psi_{1}\left(y_{1}\right)+\ldots+\psi_{r}\left(y_{r}\right)\right)+b,
$$

для всех $a, b \in \mathbb{R}$. Соотношения (8) примут вид:

$$
u^{\prime} \cdot\left(\varphi_{i}^{\prime \prime}+\psi_{i}^{\prime \prime}\right)=0 .
$$

Так как $u^{\prime} \neq 0(u \neq$ const $)$, то имеем, что $\varphi_{i}^{\prime \prime}\left(x_{i}\right)+\psi_{i}^{\prime \prime}\left(y_{i}\right)=0$ для любых $i$. Так как аргументы $x_{i}$ и $y_{i}$ независимы, то $\varphi_{i}^{\prime \prime}=-\psi_{i}^{\prime \prime}=2 c_{i}=$ const, то есть

$$
\varphi_{i}\left(x_{i}\right)=c_{i} x_{i}^{2}+b_{i} x_{i}+a_{i}, \quad \psi_{i}\left(y_{i}\right)=-c_{i} y_{i}^{2}+m_{i} y_{i}+n_{i},
$$


для всех $a_{i}, b_{i}, c_{i}, m_{i}, n_{i} \in \mathbb{R}$. Подставляя найденные функции $\varphi_{i}\left(x_{i}\right)$ и $\psi_{i}\left(y_{i}\right)$ в $(9)$, получаем

$$
u=\sum_{i=1}^{r} c_{i}\left(x_{i}^{2}-y_{i}^{2}\right)+\sum_{i=1}^{r} a_{i} x_{i}++\sum_{i=1}^{r} b_{i} y_{i}+k,
$$

для всех $a_{i}, b_{i}, c_{i}, k \in \mathbb{R}$.

б) Если $u^{\prime \prime} \neq 0$, то уравнения (6) и (7) принимают следующий вид:

$$
\begin{array}{r}
\varphi_{i}^{\prime} \cdot \psi_{j}^{\prime}-\varphi_{j}^{\prime} \cdot \psi_{i}^{\prime}=0 \quad \forall i, j, \\
\varphi_{i}^{\prime} \cdot \varphi_{j}^{\prime}+\psi_{i}^{\prime} \cdot \psi_{j}^{\prime}=0 \text { при } i \neq j .
\end{array}
$$

Из уравнений $\left(6^{\prime}\right)$ имеем $\frac{\varphi_{i}^{\prime}\left(x_{i}\right)}{\varphi_{j}^{\prime}\left(x_{j}\right)}=\frac{\psi_{i}^{\prime}\left(y_{i}\right)}{\psi_{j}^{\prime}\left(y_{j}\right)}$. Так как левая и правая части зависят от разных переменных, то эти отношения могут быть только константами, то есть $\frac{\varphi_{i}^{\prime}\left(x_{i}\right)}{\varphi_{j}^{\prime}\left(x_{j}\right)}=\frac{\psi_{i}^{\prime}\left(y_{i}\right)}{\psi_{j}^{\prime}\left(y_{j}\right)}=c_{i j}=$ const, или

$$
\varphi_{i}^{\prime}\left(x_{i}\right)=c_{i j} \varphi_{j}^{\prime}\left(x_{j}\right), \quad \psi_{i}^{\prime}\left(y_{i}\right)=c_{i j} \psi_{j}^{\prime}\left(y x_{j}\right) .
$$

Поскольку аргументы функций левых и правых частей последних уравнений независимы, то они выполняются тогда и только тогда, когда $\varphi_{i}^{\prime}\left(x_{i}\right)=a_{i}, \psi_{i}^{\prime}\left(y_{i}\right)=c_{i}, a_{i}=\mathrm{const}, c_{i}=\mathrm{const}$, откуда $\varphi_{i}\left(x_{i}\right)=a_{i} x_{i}+b_{i}$, $\psi_{i}\left(y_{i}\right)=c_{i} y_{i}+k_{i}, a_{i}, b_{i}, c_{i}, k_{i} \in \mathbb{R}$. В силу $\left(7^{\prime}\right)$ коэффициенты этих функций связаны условиями

$$
a_{i} \cdot a_{j}+c_{i} \cdot c_{j}=0, \quad i \neq j
$$

Последние условия можно переписать в виде $\frac{a_{i}}{c_{i}}=-\frac{a_{j}}{c_{j}} \forall i, j$. Полагая $\frac{a_{i}}{c_{i}}=p_{i}$, получим $p_{i}=-p_{j}, p_{i}=-p_{k}, p_{j}=-p_{k}$, а следовательно $p_{i}=0$.

Таким образом, условия (11) выполняются только если $a_{i}=c_{i}=0$, для всех $i$, т. е. функции $\varphi_{i}\left(x_{i}\right)$ и $\psi_{i}\left(y_{i}\right)$ являются постоянными. Итак, в случае $u^{\prime \prime} \neq 0$ получаем только тривиальное решение $u=$ const, то есть ткань не существует. Доказана

Теорема 1. Уравнение регулярной $(2 r+1)$-ткани, определяемой плюригармонической функиией, имеет вид (10).

2. Пусть плюригармонические функции имеют вид (3), где

$$
\varphi\left(x_{1}, \ldots, x_{r}\right)=\varphi\left(x_{i}\right), \quad \psi\left(y_{1}, \ldots, y_{r}\right)=\psi\left(y_{i}\right) .
$$

Тогда уравнения (5) примут вид:

$$
u^{\prime \prime} \cdot\left(\varphi_{x_{i}}^{\prime} \cdot \psi_{y_{j}}^{\prime}-\varphi_{x_{j}}^{\prime} \cdot \psi_{y_{i}}^{\prime}\right)=0 \quad \forall i, j
$$


а уравнения (4) -

$$
u^{\prime \prime} \cdot\left(\varphi_{x_{i}}^{\prime} \varphi_{x_{j}}^{\prime}+\psi_{y_{i}}^{\prime} \psi_{y_{j}}^{\prime}\right)+u^{\prime} \cdot\left(\varphi_{x_{i} x_{j}}^{\prime \prime}+\psi_{y_{i} y_{j}}^{\prime \prime}\right)=0 \quad \forall i, j
$$

а) Если $u^{\prime \prime}=0$, то уравнения (12) удовлетворяются, а функция $u$ является линейной:

$$
u=a\left(\varphi\left(x_{i}\right)+\psi\left(y_{i}\right)\right)+b, \quad a, b \in \mathbb{R} .
$$

При этом уравнения (13) перепишутся в виде $u^{\prime} \cdot\left(\varphi_{x_{i} x_{j}}^{\prime \prime}+\psi_{y_{i} y_{j}}^{\prime \prime}\right)=0$.

Так как $u^{\prime} \neq 0(u \neq$ const $)$, то имеем $\varphi_{x_{i} x_{j}}^{\prime \prime}+\psi_{y_{i} y_{j}}^{\prime \prime}=0$ для любых $i, j$. Так как аргументы функций $\varphi\left(x_{i}\right)$ и $\psi\left(y_{i}\right)$ независимы, то

$$
\varphi_{x_{i} x_{j}}^{\prime \prime}=-\psi_{y_{i} y_{j}}^{\prime \prime}=2 c_{i j}=\text { const, }
$$

то есть указанные функции имеют следующее строение

$$
\begin{aligned}
& \varphi\left(x_{1}, \ldots, x_{r}\right)=\sum_{i, j=1}^{r} c_{i j} x_{i} x_{j}+\sum_{i=1}^{r} a_{i} x_{i}+k \\
& \psi\left(y_{1}, \ldots, y_{r}\right)=-\sum_{i, j=1}^{r} c_{i j} y_{i} y_{j}+\sum_{i=1}^{r} m_{i} y_{i}+p
\end{aligned}
$$

где $c_{i j}, a_{i}, m_{i}, k, p \in \mathbb{R}$. Подставляя найденные функции $(15)$ в $(14)$, получаем

$$
u=\sum_{i, j=1}^{r} c_{i j}\left(x_{i} x_{j}-y_{i} y_{j}\right)+\sum_{i=1}^{r} a_{i} x_{i}+\sum_{i=1}^{r} m_{i} y_{i}+b, \quad c_{i j}, a_{i}, m_{i}, b \in \mathbb{R} .
$$

б) Если $u^{\prime \prime} \neq 0$, то из уравнений $(12)$ получим $\varphi_{x_{i}}^{\prime} \cdot \psi_{y_{j}}^{\prime}-\varphi_{x_{j}}^{\prime} \cdot \psi_{y_{i}}^{\prime}=0$ или

$$
\frac{\varphi_{x_{i}}^{\prime}\left(x_{1}, \ldots, x_{r}\right)}{\varphi_{x_{j}}^{\prime}\left(x_{1}, \ldots, x_{r}\right)}=\frac{\psi_{y_{i}}^{\prime}\left(y_{1}, \ldots, y_{r}\right)}{\psi_{y_{j}}^{\prime}\left(y_{1}, \ldots, y_{r}\right)}
$$

Так как левая и правая части зависят от разных переменных, то эти отношения могут быть только константами, то есть

$$
\frac{\varphi_{x_{i}}^{\prime}\left(x_{1}, \ldots, x_{r}\right)}{\varphi_{x_{j}}^{\prime}\left(x_{1}, \ldots, x_{r}\right)}=\frac{\psi_{y_{i}}^{\prime}\left(y_{1}, \ldots, y_{r}\right)}{\psi_{y_{j}}^{\prime}\left(y_{1}, \ldots, y_{r}\right)}=c_{i j}=\text { const. }
$$

Отсюда получим $\varphi_{x_{i}}^{\prime}=c_{i j} \varphi_{x_{j}}^{\prime}, \psi_{y_{i}}^{\prime}=c_{i j} \psi_{y_{j}}^{\prime}$.

Последние равенства означают, что функции $\varphi_{x_{i}}^{\prime}\left(x_{1}, \ldots, x_{r}\right)$ пропорциональны друг другу. 
Потому полный дифференциал функции $\varphi\left(x_{1}, \ldots, x_{r}\right)$ имеет вид:

$$
\begin{aligned}
d \varphi & =\sum_{i=1}^{r} \varphi_{x_{i}}^{\prime} d x_{i}=\varphi_{x_{1}}^{\prime} d x_{1}+\ldots+\varphi_{x_{r}}^{\prime} d x_{r}= \\
& =\varphi_{x_{1}}^{\prime} d x_{1}+\ldots+c_{1 r} \varphi_{x_{1}}^{\prime} d x_{r}=\varphi_{x_{1}}^{\prime} d\left(x_{1}+\ldots+c_{1 r} x_{r}\right) .
\end{aligned}
$$

Последнее означает, что функция $\varphi\left(x_{i}\right)$ имеет следующее строение:

$$
\varphi\left(x_{1}, \ldots, x_{r}\right)=\varphi\left(\alpha_{1} x_{1}+\ldots+\alpha_{r} x_{r}\right), \quad \alpha_{i} \in \mathbb{R} .
$$

Рассуждая аналогично для функции $\psi\left(y_{i}\right)$, получим

$$
\psi\left(y_{1}, \ldots, y_{r}\right)=\psi\left(\alpha_{1} y_{1}+\ldots+\alpha_{r} y_{r}\right), \quad \alpha_{i} \in \mathbb{R} .
$$

С учётом строения функций $\varphi\left(x_{i}\right)$ и $\psi\left(y_{i}\right)$ уравнение (16) дает:

$$
\alpha_{i} \alpha_{j}\left(u^{\prime \prime} \cdot\left(\left(\varphi^{\prime}\right)^{2}+\left(\psi^{\prime}\right)^{2}\right)+u^{\prime}\left(\varphi^{\prime \prime}+\psi^{\prime \prime}\right)\right)=0, \quad \alpha_{i} \neq 0, \quad \forall i .
$$

Последнее равенство перепишем в виде:

$$
\varphi^{\prime \prime}+\psi^{\prime \prime}=-\frac{u^{\prime \prime}}{u^{\prime}} \cdot\left(\left(\varphi^{\prime}\right)^{2}+\left(\psi^{\prime}\right)^{2}\right),
$$

и обозначим $-\frac{u^{\prime \prime}}{u^{\prime}}=g$. Тогда получим уравнение

$$
\varphi^{\prime \prime}+\psi^{\prime \prime}=g \cdot\left(\left(\varphi^{\prime}\right)^{2}+\left(\psi^{\prime}\right)^{2}\right) .
$$

Продифференцировав равенство (17) по $x_{k}$, а затем по $y_{m}$, придём к уравнению

$$
\alpha_{k} \alpha_{m} \varphi^{\prime} \psi^{\prime}\left(g^{\prime \prime}\left(\left(\varphi^{\prime}\right)^{2}+\left(\psi^{\prime}\right)^{2}\right)+2 g^{\prime}\left(\varphi^{\prime \prime}+\psi^{\prime \prime}\right)\right)=0 .
$$

Так как $\varphi$ и $\psi$ не являются постоянными, то $\varphi^{\prime} \psi^{\prime} \neq 0$. Приравнивая выражение в скобках к нулю, с учетом (17) получим уравнение

$$
g^{\prime \prime}+2 g^{\prime} \cdot g=0
$$

или $g^{\prime \prime}+\left(g^{2}\right)^{\prime}=0$. Интегрируя, получим $g^{\prime}+g^{2}=c, c \in \mathbb{R}$.

1) В случае $c=a^{2}$ решение имеет вид $g=-a \cdot \operatorname{coth} a(b-\theta)$.

2) В случае $c=-a^{2}$ решение имеет вид $g=a \cdot \tan a(b-\theta)$.

3) В случае $c=0$ решение имеет вид $g=\frac{1}{b+\theta}$.

Во всех случаях $b \in \mathbb{R}, \theta=\varphi\left(x_{i}\right)+\psi\left(y_{i}\right)$.

В первом случае при подстановке в (17) функции $g$, получим

$$
\varphi^{\prime \prime}+\psi^{\prime \prime}=-a \cdot \operatorname{coth} a(b-\theta) \cdot\left(\left(\varphi^{\prime}\right)^{2}+\left(\psi^{\prime}\right)^{2}\right) .
$$

Продифференцировав это равенство по $x_{k}$, а затем по $y_{m}$, получим

$$
2 a^{3} \alpha_{k} \alpha_{m} \varphi^{\prime} \psi^{\prime} \frac{\sinh a(b-\varphi-\psi)}{\cosh ^{3} a(b-\varphi-\psi)}=0 .
$$


Так как функции $\varphi$ и $\psi$ не являются постоянными, то отсюда находим

$$
\sinh a(b-\varphi-\psi)=0, \quad \varphi+\psi=b
$$

что невозможно, поскольку $\varphi$ и $\psi$ зависят от разных независимых аргументов.

Во втором случае получаем аналогичный результат.

В третьем случае равенство (17) примет вид

$$
\left(\varphi^{\prime \prime}+\psi^{\prime \prime}\right)(b+\varphi+\psi)=\left(\varphi^{\prime}\right)^{2}+\left(\psi^{\prime}\right)^{2} .
$$

Продифференцировав равенство (18) по $x_{k}$, а затем по $y_{m}$, получим

$$
\varphi^{\prime \prime \prime} \psi^{\prime}+\varphi^{\prime} \psi^{\prime \prime \prime}=0
$$

Отсюда $\frac{\varphi^{\prime \prime \prime}}{\varphi^{\prime}}=-\frac{\psi^{\prime \prime \prime}}{\psi^{\prime}}=-p^{2}=$ const, или

$$
\varphi^{\prime \prime \prime}+p^{2} \varphi^{\prime}=0, \quad \quad \psi^{\prime \prime \prime}-p^{2} \psi^{\prime}=0
$$

Пусть $\varphi^{\prime \prime \prime} \neq 0, \psi^{\prime \prime \prime} \neq 0$, тогда решения последних уравнений имеют следующий вид:

$$
\begin{aligned}
& \varphi\left(\sum_{i=1}^{r} \alpha_{i} x_{i}\right)=\frac{r}{p} \cos \left(p \sum_{i=1}^{r} \alpha_{i} x_{i}\right)+\frac{s}{p} \sin \left(p \sum_{i=1}^{r} \alpha_{i} x_{i}\right) \\
& \psi\left(\sum_{i=1}^{r} \alpha_{i} y_{i}\right)=\frac{g}{p} \operatorname{coth}\left(p \sum_{i=1}^{r} \alpha_{i} y_{i}\right)+\frac{h}{p} \sinh \left(p \sum_{i=1}^{r} \alpha_{i} y_{i}\right)
\end{aligned}
$$

где $r, s, g, h \in \mathbb{R}$.

Найдем функцию $u$ из уравнения $-\frac{u^{\prime \prime}}{u^{\prime}}=\frac{1}{b+\varphi\left(x_{i}\right)+\psi\left(y_{i}\right)}$ (см. выше). После интегрирования, получим $u=c_{1} \ln \left(b+\varphi\left(x_{i}\right)+\psi\left(y_{i}\right)\right)+c_{2}$, где $c_{1}=c_{2}=$ const.

Таким образом, учитывая (19), окончательно получаем

$$
\begin{aligned}
u & =c_{1} \ln \left(b+\frac{r}{p} \cos \left(p \sum_{i=1}^{r} \alpha_{i} x_{i}\right)+\frac{s}{p} \sin \left(p \sum_{i=1}^{r} \alpha_{i} x_{i}\right)+\right. \\
& \left.+\frac{g}{p} \operatorname{coth}\left(p \sum_{i=1}^{r} \alpha_{i} y_{i}\right)+\frac{h}{p} \sinh \left(p \sum_{i=1}^{r} \alpha_{i} y_{i}\right)\right)+c_{2} .
\end{aligned}
$$


Рассмотрим случай, когда $\varphi^{\prime \prime \prime}=0, \psi^{\prime \prime \prime}=0$. Тогда функции $\varphi$ и $\psi$ имеют следующее строение:

$$
\begin{aligned}
\varphi\left(\sum_{i=1}^{r} \alpha_{i} x_{i}\right) & =a_{0}\left(\sum_{i=1}^{r} \alpha_{i} x_{i}\right)^{2}+a_{1} \sum_{i=1}^{r} \alpha_{i} x_{i}+a_{2}, \\
\psi\left(\sum_{i=1}^{r} \alpha_{i} y_{i}\right) & =b_{0}\left(\sum_{i=1}^{r} \alpha_{i} y_{i}\right)^{2}+b_{1} \sum_{i=1}^{r} \alpha_{i} y_{i}+b_{2},
\end{aligned}
$$

а функция $u$ имеет вид:

$$
\begin{aligned}
u=c_{1} \ln \left(b+a_{0}\left(\sum_{i=1}^{r} \alpha_{i} x_{i}\right)^{2}+\right. & a_{1} \sum_{i=1}^{r} \alpha_{i} x_{i}+ \\
& \left.+b_{0}\left(\sum_{i=1}^{r} \alpha_{i} y_{i}\right)^{2}+b_{1} \sum_{i=1}^{r} \alpha_{i} y_{i}\right)+c_{2} .
\end{aligned}
$$

Таким образом, доказана

Теорема 2. Уравнение регулярной ткани $W(r, r, 2 r-1)$, определяемой плюригармонической функцией, имеет вид (20) или (21).

\section{ЛитерАТУРА}

[1] В. Бляшке. Введение в геометрию тканей. М., Физматгиз, 1956.

[2] Б. В. Шабат. Введение в комплексный анализ. М., Наука, 1985.

[3] А. М. Шелехов. О «шестиугольных» решениях некоторых уравнений в частных производных. Proceedings of the International Geometry Center, 10(2):47-55, 2017.

[4] А. М. Шелехов, В. Б. Лазарева, А. А. Уткин. Криволинейные три-ткани. Тверь, 2013.

Поступила в редакцию 18 сентября 2018, принята к печати 2 ноября 2018.

Пиджакова Любовь Михайловна

ТВЕРСКОЙ ГОСУДАРСТВЕННЫЙ ТЕХНИЧЕСКИЙ УНИВЕРСИТЕТ

Email: lpidjhacova@mail.ru

Шелехов Александр Михайлович

МОСКОВСКИЙ ПЕДАГОГИЧЕСКИЙ ГОСУДАРСТВЕННЫЙ УНИВЕРСИТЕТ

Email: amshelekhov@rambler.ru 